BULLETIN OF THE

AMERICAN MATHEMATICAL SOCIETY

Volume 80, Number 2, March 1974

\title{
PSEUDO-INVERSES OF OPERATORS
}

BY J. J. KOLIHA

Communicated by Robert Bartle, August 13, 1973

1. Let $X$ and $Y$ be complex Banach spaces, $A$ a bounded linear operator from $X$ to $Y$. If the null space $N(A)$ and the closed range $R(A)^{-}$possess closed complementary subspaces $U$ in $X$ and $V$ in $Y$ respectively, the pseudo-inverse $A^{\dagger}$ of $A$ relative to $(U, V)$ is defined as the linear extension of $(A \mid U)^{-1}$ to $D\left(A^{\dagger}\right)=R(A)+V$ with the null space $N\left(A^{\dagger}\right)=V$. (This is a generalization to Banach space of the standard pseudo-inverse of a Hilbert space operator (cf. [8]). If $R(A)$ is closed, the definition agrees with the ones given in [1] and [7]. In this case $A^{\dagger}$ is defined and bounded on all of $Y$.) If $U=R(B)^{-}$and $V=N(B)$ for some bounded linear operator $B: Y \rightarrow X, A^{\dagger}$ will be called the pseudo-inverse of $A$ relative to $B$, written $A^{\dagger B}$. Proposition 6 of [6] leads to the following result.

Theorem 1. Suppose $A: X \rightarrow Y$ and $B: Y \rightarrow X$ are bounded linear operators such that (a) $Y=R(A)^{-} \oplus N(B)$, (b) the operator $T=I-B A$ is strongly power convergent $\left(\left\{T^{n}\right\}\right.$ converges strongly). Then $A^{\dagger B}$ exists and is represented by

$$
A^{\dagger B} y=\sum_{n=0}^{\infty}(I-B A)^{n} B y,
$$

where the series converges in norm iff $y \in R(A)+N(B)$.

When $T$ in Theorem 1 is uniformly power convergent $\left(\left\{T^{n}\right\}\right.$ converges uniformly), then $R(A)$ is closed, (1) converges uniformly, and $A^{\dagger B}$ is defined and bounded on all of $Y$. In the case that $A$ is an operator between Hilbert spaces, and $B=\alpha A^{*}$ with $0<\alpha<2\|A\|^{-2}$, Theorem 1 gives the well-known representation of the standard Hilbert space pseudo-inverse [2], [7], [8].

2. Let $A: X \rightarrow Y$ be a bounded linear operator between Banach spaces. A bounded linear operator $B: Y \rightarrow X$ is called a pseudo-adjoint of $A$ if

$$
X=N(A) \oplus R(B)^{-}, \quad Y=R(A)^{-} \oplus N(B),
$$

AMS (MOS) subject classifications (1970). Primary 47A99, 47A50; Secondary 47A10, $65 \mathrm{~J} 05$.

Key words and phrases. Pseudo-inverse of an operator, partial inverse, series expansion, power convergent operator, pseudo-adjoint, spectrum of an operator.

Copyright @ American Mathematical Society 1974 
and if there exists a real function $h$ on $R(B)$ such that the operator $T=I-\alpha B A$ (with a suitable $\alpha$ ) satisfies

$$
\begin{gathered}
0<h(x) \leqq\left(\|x\|^{2}-\|T x\|^{2}\right)\|x\|^{-4} \quad(x \neq 0), \quad h(0)=0, \\
h(T x) \geqq h(x) .
\end{gathered}
$$

The adjoint $A^{*}$ of an operator $A$ between Hilbert spaces is its pseudoadjoint $\left(h(x)=\alpha\left(2-\alpha\|A\|^{2}\right)\left\|\left(A^{*}\right)^{\dagger} x\right\|^{-2}, 0<\alpha<2\|A\|^{-2}\right)$. An idempotent operator $A$ on a Hilbert space is its own pseudo-adjoint $(h(x)=$ $\left.\alpha(2-\alpha)\|x\|^{-2}, 0<\alpha<2\right)$.

THEOREM 2. Let $B$ be a pseudo-adjoint of $A$ (with $\alpha=1$ for simplicity). Then $T=I-B A$ is a strongly power convergent operator. For each $x \in R(B)^{-}$, $\left\|T^{n} x\right\| \rightarrow 0$ monotonically, and

$$
\left\|T^{n} x\right\|^{2} \leqq\|x\|^{2}\left(1+n h(x)\|x\|^{2}\right)^{-1} \quad \text { if } x \in R(B) .
$$

Proof is based on the inequality $\left\|T^{n+1} x\right\|^{2} \leqq\left\|T^{n} x\right\|^{2}-h(x)\left\|T^{n} x\right\|^{4}$ derived from (3) and (4) and the formula (4.11) of [8]. The next theorem generalizes Theorem 2(a) and (b) of [8] to operators between Banach spaces.

THEOREM 3. Let $B$ be a pseudo-adjoint of $A$ (with $\alpha=1)$. Then

$$
\left\|\sum_{k=0}^{n}(I-B A)^{k} B y-A^{\dagger^{B}} y\right\|^{2} \leqq\left\|A^{\dagger B} y\right\|^{2}\left(1+n h\left(A^{\dagger B} y\right)\left\|A^{\dagger B} y\right\|^{2}\right)^{-1}
$$

whenever the $R(A)^{-}$component of $y$ in $Y=R(A)^{-} \oplus N(B)$ lies in $R(A B)$. $M o r e o v e r$, the left-hand side of (5) converges monotonically to 0 for each $y \in R(A)+N(B)$.

3. Let $A: X \rightarrow Y$ be a bounded linear operator, and let $U$ be a complement of $N(A)$ in $X$. The operator $A^{\partial}=(A \mid U)^{-1}$ will be called the partial inverse of $A$ relative to $U$.

TheOREM 4. Let $A: X \rightarrow Y$ and $B: Y \rightarrow X$ be bounded linear operators, with $B$ bijective and such that $T=I-B A$ is strongly power convergent. Then $A$ has the partial inverse $A^{\partial}$ relative to $U=R(B A)^{-}$, represented by

$$
A^{\partial} y=\sum_{n=0}^{\infty}(I-B A)^{n} B y,
$$

where the series converges iff $y \in R(A)$.

When the convergence of $\left\{T^{n}\right\}$ in the preceding theorem is uniform, $R(A)$ is closed, $A^{\partial}$ bounded, and the series (6) converges uniformly on bounded sets of $R(A)$. 
Both Theorems 1 and 4 can be applied to the approximate solution of the linear equation $A x=y$ by means of the Picard iterations

$$
x_{n+1}=(I-B A) x_{n}+B y \quad\left(x_{0} \text { given }\right) .
$$

In either case, if $y \in R(A),\left\{x_{n}\right\}$ converges in norm to the solution $x=P x_{0}+$ $A^{\partial} y$ of $A x=y$, where $P x_{0}$ is the $N(A)$ component of $x_{0}$ in $X=N(A) \oplus$ $R(B A)^{-}$. (In the case of Theorem $1, A^{\partial} y=A^{\dagger B} y$ and $R(B A)^{-}=R(B)^{-}$.)

4. The strong power convergence of the operator $T: X \rightarrow X$ is the main hypothesis of Theorems 1 and 4. Various conditions for power convergence have been given in [2], [3], [4], [5]. It was shown in [5] that $T$ is uniformly power convergent iff $\sigma(T)-\{1\}$ lies in the open unit disc and 1 is a pole of $(\lambda I-T)^{-1}$ of order $\leqq 1(\sigma(T)$ denotes the spectrum of $T)$. The following three results can be obtained from this theorem.

THEOREM 5. Suppose $R(I-T)$ is closed and the continuous spectrum of $T$ does not meet the unit circle. Then the weak, strong and uniform power convergence of $T$ are all equivalent.

The proof is based on the decomposition $T=T_{0} \oplus T_{1}$ of a weakly power convergent $T$, where $T_{0}=I \mid N(I-T)$ and $T_{1}=T \mid R(I-T)^{-}[6]$.

THEOREM 6. Let $T$ be power bounded, $R(I-T)$ closed, and let $I-T$ have finite descent. Then $T$ is uniformly power convergent iff $\sigma(T)-\{1\}$ does not meet the unit circle.

To prove Theorem 6 , we show that $N\left((I-T)^{2}\right)=N(I-T)$ under the assumptions of the theorem.

The following result is a consequence of Theorems 5 and 6 .

COROLlaRY 1. Suppose that $T$ is power bounded and $f(T)$ compact, where $f$ is a complex function analytic in an open neighborhood of $\sigma(T)$ with no zeros on $\sigma(T)-\{0\}$ such that (a) $|f(\lambda)|<1$ if $|\lambda|<1$, (b) $f(1)=1$, and (c) $f^{\prime}(1) \neq 0$. Then $T$ is weakly (=strongly=uniformly) power convergent iff $\sigma(T)-\{1\}$ does not meet the unit circle.

The next three theorems give sufficient conditions of the Stein type (cf. [5]) for power convergence of Hilbert space operators. In the sequel, $A, T$ and $W$ are bounded linear operators on a Hilbert space $H$.

THEOREM 7. Let $A=A^{*}$, and $A-T^{*} A T$ be positive definite on $R(I-T)^{-}$. Then the following conditions are equivalent: (i) $\left\{T^{n}\right\}$ converges uniformly, (ii) $\left\{T^{n}\right\}$ converges strongly, (iii) $A$ is positive definite on $R(I-T)^{-}$. 
TheOREM 8. Suppose the identity

$$
A-T^{*} A T=\left(I-T^{*}\right) W(I-T)
$$

holds with $A$ and $W$ positive definite on $H$. Then $T$ is strongly power convergent.

THEOREM 9. Suppose the identity (8) holds with $A$ and $W$ positive definite on $R(I-T)^{-}$. If $I-T$ is an operator of finite descent, then $T$ is uniformly power convergent.

We outline the proof of the last theorem. We establish $N(I-T) \cap$ $R(I-T)^{-}=\{0\}$ by showing that $(A x, x)=(A x, h)$ for each $x=(I-T) u+h$. Hence $X=N(I-T) \oplus R(I-T)$ with $R(I-T)$ closed. The rest is easy.

\section{REFERENCES}

1. F. J. Beutler, The operator theory of the pseudo-inverse. I: Bounded operators, J. Math. Anal. Appl. 10 (1965), 451-470. MR 31 \#3865a.

2. F. E. Browder and W. V. Petryshyn, The solution by iteration of linear functional equations in Banach space, Bull. Amer. Math. Soc. 72 (1966), 566-570. MR 32 \#8155a.

3. F. Gilfeather, Asymptotic convergence of operators in Hilbert spaces, Proc. Amer. Math. Soc. 22 (1969), 69-76. MR 40 \#773.

4. - The structure and asymptotic behavior of polynomially compact operators, Proc. Amer. Math. Soc. 25 (1970), 127-134. MR 41 \#2440.

5. J. J. Koliha, Convergent and stable operators and their generalization, J. Math. Anal. Appl. 43 (1973) (to appear).

6. - Ergodic theory and averaging iterations, Canad. J. Math. 25 (1973), 14-23.

7. M. Z. Nashed, Generalized inverses, normal solvability and iteration for singular operator equations, Nonlinear Functional Analysis and Applications (Proc. Advanced Sem., Math. Res. Center, Univ. of Wisconsin, Madison, Wis., 1970), Academic Press, New York, 1971, pp. 311-359. MR 43 \#1003.

8. D. W. Showalter and A. Ben-Israel, Representation and computation of the generalized inverse of a bounded linear operator between Hilbert spaces, Atti Accad. Naz. Lincei Rend. Cl. Sci. Fis. Mat. Natur. (8) 48 (1970), 184-194. MR 42 \#8310.

Department of Mathematics, University of Melbourne, Parkville, Victoria 3052, Australia 